\title{
PUBLIC HEALTH AND HEALTH CARE
}

UDC 616.98:578.834.1-082.4

\section{Operating experience of a multidisciplinary medical hospital under the conditions of reprofiling to receive patients with the new coronavirus infection COVID-19}

\author{
A. E. Nikitin, Yu.A.Shikhova, I. V. Velichko \\ Central Clinical Hospital of the Russian Academy of Sciences, \\ 1a, Litovsky bul., Moscow, 117593, Russian Federation
}

For citation: Nikitin A. E., Shikhova Yu. A., Velichko I. V. Operating experience of a multidisciplinary medical hospital under the conditions of reprofiling to receive patients with the new coronavirus infection COVID-19. Vestnik of Saint Petersburg University. Medicine, 2021, vol. 16, issue 3, pp. 203-210. https://doi.org/10.21638/spbu11.2021.307

The purpose of our work was to increase the efficiency of medical care provided to patients with the new coronavirus infection COVID-19 who were admitted to a multidisciplinary hospital, based on a retrospective analysis of the work carried out to reorganise material-technical and personnel support, organise operations in an unfavourable epidemiological situation in the period from 16.05-06.11.2020. During the specified work period, data on 677 hospitalised patients were analysed. The practical experience of the FGBUZ CCH RAS demonstrated the feasibility of a quick response to unfavourable development of the epidemiological situation associated with the spread of COVID-19 via solving the problem of reorganising a multidisciplinary hospital, taking into account the implementation of organisational measures that provide the material and technical basis for the diagnosis and treatment of patients and measures aimed at preventing the spread of infection among medical personnel and patients. Our data confirmed that complications related to infection with COVID-19 and which led to the need to use intensive care measures were more typical for people in the older age group: with an average age of $57 \pm 6$ years for men and $64 \pm 7$ years for women that were admitted to the hospital. A set of measures to ensure the safety of personnel involved in providing medical care to patients with the new coronavirus infection minimises the risks of personnel infection. Keywords: new coronavirus infection COVID-19, conversion of a multidisciplinary hospital, unfavorable epidemiological situation, personnel safety, prevention of risks of infection.

(C) St. Petersburg State University, 2021 


\section{Introduction}

The significance of the provision of timely medical care to the population, as well as the protection of medical personnel in the context of the COVID-19 pandemic in Russia, is associated with alarming statistics on the number of cases in the country: as of $13 / 11 / 2020$, the number of cases is 1858568 , recovered -1388168 , deaths $-32037(1.7 \%$ of the number of cases) $)^{1}$.

The pandemic of the new coronavirus infection has affected not only the healthcare system but all spheres of society's life - economic, social, political and spiritual.

The search for ways to overcome the pandemic has contributed to the intensification of scientific research in almost all subject areas. As a result of a search for scientific publications on the topic of a pandemic of the new coronavirus infection only on the largest Russian information and analytical portal in the field of science, technology, medicine and education - Scientific Electronic Library (http://www.elibrary.ru) by the search query "COVID-19" (as of 13.11.2020), 5798 scientific articles were found that were published in 2020, of which 52 articles were in the thematic section "Medicine and Healthcare" and considered the issues of reorganising domestic healthcare system in the context of a pandemic caused by the single-stranded shell SARS-CoV-2 virus ${ }^{2}$.

At the same time, primarily the recommendations of the World Health Organization and, first of all, the experience of the People's Republic of China [1-3] are considered; world data on the aetiology, pathogenesis, clinical features of coronavirus infection COVID-19 are summarised [4-8]; the course of the epidemic process in conjunction with the ongoing anti-epidemic measures in the Russian Federation is studied [9].

Additionally, despite the extensive anti-epidemic measures, the successful development of vaccines, the issue of the COVID-19 pandemic remains tense, which, in turn, emphasises the need to adjust the strategy and tactics of treating the new coronavirus infection, to improve the existing methodological approaches to its prevention, diagnosis and treatment recommended by the Ministry of Healthcare of the Russian Federation ${ }^{3}$.

In the spring of 2020 period of the pandemic, new data on safety and risk of pharmacotherapy used in treatment [10], the role of age, concomitant diseases and the activity of the renin-angiotensin-aldosterone system in the manifestations of COVID-19 have already been obtained and published [11].

The generalisation of data on the provision of medical care to and treatment of patients with COVID-19 provided in the article by Zhirnov (2020) based on 55 foreign sources allows to apply a differentiated approach to treatment regimens at the early (etiotropic) and late (pathogenetic) stages of the disease already today using, at an early stage, chemotherapeutic agents aimed at various viral targets: inhibitors of viral RNA polymerase, inhibitors of the viral protease Mpro, inhibitors of proteolytic activation of viral

\footnotetext{
${ }^{1}$ About confirmed cases of the new coronavirus infection COVID-2019 in Russia [electronic resource]. Website of the Federal Service for Surveillance on Consumer Rights Protection and Human Welfare. Available at: https://www.rospotrebnadzor.ru/ (accessed: 13.11.2020). (In Russian)

${ }^{2}$ Website of the scientific electronic library [electronic resource]. Available at: http://www.elibrary.ru (accessed: 13.11.2020).

3 Temporary guidelines "Prevention, diagnosis and treatment of new coronavirus infection (COVID-19)" of the Ministry of Healthcare of the Russian Federation. Version 7 dated 06.03.2020. [Electronic resource]. Available at: http://edu.rosminzdrav.ru/fileadmin/user_upload/specialists/COVID-19/ MR_COVID-19_v7.pdf (accessed: 13.11.2020). (In Russian)
} 
protein S, which facilitates the virus entry into the target cell, inhibitors of viral deproteinisation in cell endosomes, preparations of exogenous interferon, natural preparations and recombinant virus neutralising antibodies; and, at the second stage, when the multiplication of the virus declines and the threatening pathological processes of excessive inflammation, acute respiratory distress syndrome, pulmonary tissue edema, hypoxia dominate, the use of pathogenetic agents, such as extracorporeal blood oxygenation, detoxification, anti-inflammatory and antibacterial therapeutic measures and activities are most important [12].

For stable and effective work of medical organisations during a pandemic, another important consideration is to ensure the safety of and prevent the spread of infection among medical workers and ensure their social protection [13].

In this regard, consideration and exchange of experience in medical organisations' work in the conditions of admitting patients with COVID-19 for treatment is a necessary component of improving the provision of high-quality and timely care to patients, which determined the relevance of our work [4].

The aim of the study was to increase the efficiency of the provision of medical care to patients with COVID-19 admitted to a multidisciplinary hospital based on a retrospective analysis of the work carried out on during the reorganisation and the process of ensuring the hospital's functioning in an unfavourable epidemic situation.

To achieve this goal, the following main tasks were solved step-by-step: 1) reorganising of a multidisciplinary hospital during an unfavourable epidemiological situation to admit patients with COVID-19 while ensuring the safety of medical personnel; 2) improving the diagnosis of the disease and its complications, as well as the tactics of treating patients with COVID-19 with varying severity of the disease; 3) analysing statistical data on patients and hospital work for the period of admission of patients with COVID-19; 4) summarising the hospital's experience in providing medical care to patients with COVID-19.

\section{Methodology}

The study used data on the medical activities of the Federal State Budgetary Institution of Healthcare Central Clinical Hospital of the Russian Academy of Sciences (FGBUZ CCH RAS) in the period from 16.04.2020. to 06.11.2020, which, in accordance with the Order of the Government of the Russian Federation dated 02.04.2020 No. 844-r from 13.04.2020, was re-profiled to provide medical care to patients with the new coronavirus infection COVID-19, and, in accordance with the Letter of the Founder of the FGBUZ $\mathrm{CCH}$ RAS - the Ministry of Science and Higher Education dated 05.06.2020 No. MN9/994-AM "On the coordination of the phased restoration of the organisation's core activities," stopped hospitalisation of patients with the new coronavirus infection COVID-19 or with suspicion of it.

To reorganise the hospital to ensure the provision of medical care to patients with the new coronavirus infection COVID-19, the experience of infectious diseases hospitals and previously reorganised hospitals, as well as the recommendations of the Ministry of Healthcare of the Russian Federation that were existing at that time, were used.

During the period of admission of patients with the new coronavirus infection COVID-19, the following data on 677 hospitalised patients were analysed: age and sex composition of patients, including those in the intensive care unit, the average length of stay in 
the hospital, the degree of lung damage (based on CT) and the proportion of the use of artificial lung ventilation, the number and structure of lethal outcomes of the disease.

The proportion of medical workers who became ill (infected) with the new coronavirus infection caused by the COVID-19 virus was determined.

\section{Results}

FGBUZ CCH RAS is a multidisciplinary medical institution, which includes inpatient departments of therapeutic and surgical profiles, as well as a widely presented diagnostic base. Despite the vast availability of all the necessary equipment for work in "peacetime", the transition to the operating mode of an infectious diseases hospital required significant changes in the structure of the institution, in its mode of operation, logistics and routing of the flow of patients and staff, as well as the implementation of measures to ensure the safety of life and health of medical personnel.

To solve the first task - the reorganisation of a multidisciplinary hospital during an unfavourable epidemiological situation while ensuring the safety of medical personnel first of all, to prevent the infection from leaving the zone where patients were located, it was necessary to organise a sanitary checkpoint with a system of locks, a separate exit, changing rooms for personnel, sanitary rooms and showers, areas for putting on personal protective equipment. In the shortest possible time, design and implementation documentation was prepared, a sanitary checkpoint with the necessary set of premises was organised in the hospital's basement.

For the work of the administrative staff, auxiliary services, temporary modular structures were erected in the immediate vicinity of the medical building.

For effective functioning in the mode of an infectious diseases hospital, the hospital departments were reorganised, six infectious diseases departments were formed to classify the patients by the severity of the disease. To ensure patients' safety, to reduce the spread of infection, a decision has been implemented on their single placement based on the principle of boxed wards.

Medical personnel have been trained on the portal of continuing medical education of the Ministry of Healthcare of the Russian Federation and received certificates in various aspects of providing medical care for the new coronavirus infection COVID-19. In addition, classes were held on compliance with sanitary and epidemiological measures when working with infectious patients. Most of the employees (65\%) acquired experience in providing medical care to patients with coronavirus pneumonia at the Scientific Research Institute of first-aid named after Sklifosovsky in Moscow.

An important component in protecting personnel from infection was the full provision of employees with protective equipment and underwear, footwear, additional fixation of clothing items, and care and hygiene products. The need was calculated based on the necessity to change personal protective equipment (PPE) once every 6-8 hours.

Throughout the entire work period in the conditions of the COVID hospital, experienced medical workers dressed the personnel entering the "redzone". Detailed visual information with the stages of putting on and taking off PPE was posted on the premises. Afterwards, for the rational use of personnel and to reduce the risk of infection, the removal of PPE was carried out under remote video control.

The hospital premises were disinfected with the recommended disinfectants regularly, following the established requirements and with the required frequency. 
Delivery of medicines, medical equipment, parcels and transfers was carried out through a system of gateways.

To minimise the risk of contamination, radios were used to communicate with personnel working in the "redzone". Medical records in the "redzone" were kept in electronic form, with the provision of contactless access in the "green zone" over a computer network. An expert group was organised in the "green zone" to work with medical documentation (registration of case histories, processing of statistical information, and current quality examination).

At the entrance and exit from the "redzone", the employees underwent contactless thermometry. A mobile laboratory unit was deployed in which hospital staff gave weekly swabs from the oropharynx and nasopharynx for subsequent detection of SARSCoV-2 RNA by PCR and ELISA for detecting antibodies to SARS-CoV-2. Thus, all employees were aware of their state of health, and the risk of spreading infection within the team was minimised.

In addition to complying with safety measures in accordance with sanitary and epidemic requirements, during the reorganisation process, it became necessary to reduce contacts of employees with people from risk groups, including family members; staff needed psycho-emotional recovery and good rest after heavy work shifts. In this regard, it was decided to place the personnel in a nearby hotel complex with 2 hot meals a day and organise a transfer to work and back.

When solving the second task - improving diagnostics and treatment tactics for patients with COVID-19 - we relied on the available temporary Methodological recommendations for the prevention, diagnosis and treatment of new coronavirus infection COVID-19 of the Ministry of Healthcare of the Russian Federation4, algorithms and treatment regimens were updated as concurrently with the recommendations. Medical care was provided to patients depending on the severity of the condition, taking into account complications and concomitant diseases.

One of the most important tasks in providing medical care to patients and ensuring their safety was to correctly organise the allocation of incoming patients at the stage of the admission department and their further routing. Thanks to pre-prepared checklists for patient interviews, informed consent templates, standardised referral forms for tests, the average time a patient spent in the admission department was 10-15 minutes. After that, the patient was sent to the CT department. The duration of the patient's stay in the department of radiation diagnostics did not exceed 5-7 minutes.

Our practice of providing medical care has confirmed that computer tomography (CT) plays the most significant role in diagnosing coronavirus pneumonia. The main radiological signs that characterise COVID-19 are the presence of a zone of reduced airiness of the pulmonary parenchyma of the "ground glass" type with or without consolidation areas. The nature of the spread of the inflammatory process depends on the stage of development of the disease and its severity, but most often, it is polysegmental. Additionally, in patients with coronavirus infection, adenopathy of the intrathoracic lymph nodes caused by reactive changes can be determined.

In a reorganised institution, CT was performed for all patients upon admission, the degree of lung damage, the degree of involvement of the lung parenchyma in the inflam-

${ }^{4}$ Temporary guidelines "Prevention, diagnosis and treatment of new coronavirus infection (COVID-19)" of the Ministry of Healthcare of the Russian Federation. Version 7 dated 06.03.2020. 
matory process was determined, followed by a decision on the further routing of the patient depending on the severity of the condition and the clinical profile.

As a result of introduced marking of patients using bracelets of different colours depending on the severity of the condition based on the results of CT diagnostics was based on the ward layout, reflecting the presence of an oxygen point, proximity to a medical post (for moderate and severe patients requiring constant visual control), the patient was assigned to the appropriate ward in the shortest time.

The organisation of a linear infectious diseases department for critically ill patients on the same floor as the intensive care unit also reduced the time spent transporting patients.

To monitor the effectiveness of patients' treatment, dynamic CT control was carried out at the frequency recommended by the treatment standards for new coronavirus infection.

During the work of the Central Clinical Hospital of the Russian Academy of Sciences as an infectious hospital, several emergency operations were performed, such as the surgical resolution of acute intestinal obstruction and myocardial revascularization in acute myocardial infarction.

Additionally, during the period of work with the new coronavirus infection, the pathological service did not stop its functioning. The handling of biomaterials obtained from infected patients, the autopsy of the deceased required special conditions for the protection of specialists, regulations for transporting, storing and subsequent burial of bodies, all of which were provided for in the process of reorganisation.

The solution to the third task of the research - analysis of statistical data on patients and the work of the hospital of the FGBUZ CCH RAS for the period of admission of patients with COVID-19 showed that out of 677 patients admitted to the hospital, by gender and age, 321 patients ( $47.3 \%$ of all hospitalised) were men (average age $-57 \pm 6$ years), 356 patients $(52.7 \%$ of all hospitalised) were women (average age $-64 \pm 7$ years). Patients spent 8822 days in the hospital; the average time spent in the hospital was 13 bed-days.

There were 115 patients in the intensive care unit, which is $17 \%$ of all hospitalised patients. At the same time, 73 patients in the intensive care unit $(63.5 \%$ of those admitted to intensive care) were men with the average age of 66 years \pm 4 years; 42 patients $(36.5 \%)$ were women, the average age was $70 \pm 5$ years.

The average age of patients in intensive care was $68 \pm 5$ years. The average length of patients stay in the intensive care unit was 11 bed-days. At the same time, $64 \%$ of patients from the total number of those admitted to intensive care had lung damages of more than $75 \%$ (CT-3; CT-4), 66 patients (57\% of those who were in intensive care; $9.7 \%$ of all admitted) were transferred on the artificial lung ventilator. 6 people were extubated (9\% of those assigned to artificial lung ventilation). 9 patients were transferred to other medical organisations ( $8 \%$ of those in intensive care).

Upon the completion of receiving medical care in the hospital, out of 677 patients were discharged home for follow-up care on an outpatient basis - 516 people $(76.2 \%)$; transferred to other medical organisations - 97 patients $(14.3 \%), 4$ patients refused treatment $(0.6 \%)$. The number of deaths is 60 (8.9\%). Of the 60 deaths, 58 patients $(97 \%$ of all deaths; $8.6 \%$ of those admitted) were with the confirmed new coronavirus infection caused by the COVID-19 virus, of which 36 patients $(60 \%$ of all deaths; $5.3 \%$ of admissions) had U07.1 Coronavirus infection caused by COVID-19 virus (virus identi- 
fied) as the main diagnosis. The average age of patients with fatal outcome was 76 years \pm 3 years.

During the entire work period, 474 medical workers were involved in providing medical care, of which 175 are doctors, 201 are nurses, and 98 are junior medical personnel. Of those infected with a new coronavirus infection caused by the COVID-19 virus - $38 \mathrm{em}-$ ployees ( $8 \%$ of the total number of medical workers), including doctors -4 , nurses -23 , junior medical personnel -11 , no severe cases of the disease among employees were registered.

The solution to the fourth task - a generalisation of the hospital's experience in providing medical care to patients with COVID-19 took place continuously in the process of daily work. The team of the Central Clinical Hospital of the Russian Academy of Sciences searched for new methods of diagnosis and treatment. In particular, a study was conducted on the feasibility of using radionuclide diagnostic methods to assess the effectiveness of treatment of patients with the new coronavirus infection. The result of the work was a conclusion on the feasibility of using molecular imaging methods such as positron emission tomography and single-photon emission tomography for the diagnosis of inflammatory cardiovascular complications in patients with confirmed COVID-19.

\section{Discussion}

The practical experience of the FGBUZ CCH RAS showed the feasibility of a quick response to the unfavourable development of the epidemiological situation associated with the spread of COVID-19 by solving the problem of reorganising a multidisciplinary hospital, taking into account the implementation of organisational measures that provide the material and technical basis for the diagnosis and treatment of patients and measures aimed at preventing the spread of infection among medical personnel and patients $[14 ; 15]$.

Our data confirmed that complications associated with infection with COVID-19 that led to the need for intensive care unit measures are more typical for people in the older age group: with an average age of men of $57 \pm 6$ years and women of $64 \pm 7$ years old admitted to the hospital; the average age patients admitted to the intensive care unit was $66 \pm 4$ years in men; in women $70 \pm 5$ years. At the same time, $64 \%$ of the total number of patients admitted to the intensive care unit had lung damage of more than $75 \%$ (according to the results of CT-3; CT-4), 66 patients (57\% of the number of persons who were in intensive care, or $9.7 \%$ of all admitted) were transferred to artificial lung ventilation. Data coincide with data of other authors [16].

\section{Conclusions}

A set of measures to ensure the safety of personnel involved in providing medical care to patients with the new coronavirus infection minimises the risks of personnel infection.

Our practical experience in reorganising a multidisciplinary institution can be used in the future when working both with patients infected with COVID-19 and in conditions of a worsening epidemic situation with infections with similar spread routes and mechanisms of influence on the body and tactics of treating infected patients. 


\section{References}

1. Report on the joint technical mission of WHO and the People's Republic of China on the problem of the new coronavirus infection COVID-2019 (extracts). Epidemiology and Vaccine Prevention, 2020, vol. 19, no. 1, pp. 98-99. (In Russian)

2. Voskanyan Y., Shikina I., Kidalov F., Davidov D. Medical Care Safety - Problems and Perspectives. Antipova T. (eds) Integrated Science in Digital Age. ICIS 2019. Lecture Notes in Networks and Systems, vol. 78. Springer, Cham. https://doi.org/10.1007/978-3-030-22493-6_26

3. Kunkel M.L., Khaivkhan K., Elemisov Sh.K., Seraliev Zh.M. Classification of common cases of COVID-2019 coronavirus infection in the PRC. Scientific Horizons, 2020, no. 4 (32), pp. 164-172. (In Russian)

4. Kolodkina E. V., Bakulina E. A., Bekker E. D. Etiology of coronavirus infection. Medical education today, 2020, no. 3 (11), pp. 157-164. (In Russian)

5. Kolodkina E. V., Latyshko O. V. Pathogenesis of coronavirus infection. Medical education today, 2020, no. 3 (11), pp. 165-173. (In Russian)

6. Sannikova N.R., Teterina A.D., Zheleznova A.D. Clinical features of coronavirus infection COVID-19. Medical education today, 2020, no. 3 (11), pp. 181-190. (In Russian)

7. Prilutsky A.S. Coronavirus disease 2019. Part 1: characteristics of the coronavirus, epidemiological features. Bulletin of hygiene and epidemiology, 2020, vol. 24, no. 1, pp. 77-86. (In Russian)

8. Prilutsky A.S. Coronavirus disease 2019. Part 2: clinic, diagnosis, treatment, prevention. Bulletin of Hygiene and Epidemiology, 2020, vol. 24, no. 1, pp. 87-101. (In Russian)

9. Kutyrev V. V., Popova A. Yu., Smolensky V. Yu., Ezhlova E. B., Demina Yu. V., Safronov V. A., Karnaukhov I. G., Ivanova A. V., Scherbakova S. A. Epidemiological features of the new coronavirus infection (Covid-19). Message 2: Features of the course of the epidemic process of covid-19 in conjunction with the ongoing anti-epidemic measures in the world and the Russian Federation. Problems of especially dangerous infections, 2020, no. 2, pp. 6-12. (In Russian)

10. Romanov B. K. Coronavirus infection COVID-2019. Safety and risk of pharmacotherapy, 2020, vol. 8, no. 1, pp. 3-8. (In Russian)

11. Mareev Y.V., Mareev V.Y. The role of age, comorbidities and the activity of the renin-angiotensinaldosterone system in the manifestations of COVID-19. Effects of ACE inhibitors of angiotensin receptor blockers. Cardiology, 2020, vol. 60, no. 4, pp. 4-9. (In Russian)

12. Zhirnov O. P. Molecular targets in chemotherapy for coronavirus infection. Biochemistry, 2020, vol. 85, no. 5, pp. 611-619. (In Russian)

13. Sevastianov M. A., Bondarev S. A., Vladimirova O.N., Bozhkov I. A. Occupational diseases of medical workers with a new coronavirus infection - medical and social assistance and social protection. National Health, 2020, no. 3, pp. 32-39. (In Russian)

14. Kopytenkova O., Shilova E. Behavioral audit of safety as a tool for decreasing occupational health and safety risk of healthcare providers. Vestnik of Saint Petersburg University, Medicine, 2020, vol. 15, no. 1, pp. 85-89. https://doi.org/10.21638/spbu11.2020.109 (In Russian)

15. Voskanyan Y., Shikina I., Kidalov F., Andreeva O., Makhovskaya T. Impact of Macro Factors on Effectiveness of Implementation of Medical Care Safety Management System. Antipova T. (ed.) Integrated Science in Digital Age 2020. ICIS 2020. Lecture Notes in Networks and Systems, vol. 136. Springer, Cham. https://doi.org/10.1007/978-3-030-49264-9_31

16. Silaev B. V., Vechorko V.I., Protsenko D.N., Averkov O.V., Khalikova E. Yu. Minimum requirements for rescuit beds and respiratory equipment in institutions refined for treatment of the new coronavirus infection COVID-19. Article. Annals of Critical Care, 2020, no. 2, pp. 34-40. https://doi. org/10.21320/1818-474X-2020-2-34-40 (In Russian)

Received: July 23, 2021 Accepted: September 2, 2021

Authors' information:

AlexeyE. Nikitin - MD, Professor; glavvrach@ckbran.ru

Yulia A. Shikhova - MD; u.shikhova@ckbran.ru

Inna V. Velichko - MD; velichko@ckbran.ru 